\title{
Lidil
}

Revue de linguistique et de didactique des langues

$45 \mid 2012$

Pratiques de formation à la lecture-écriture des adultes en parcours d'insertion

\section{Étude sur les usages des référentiels dans la formation linguistique de base}

Véronique Leclercq et Anne Vicher

\section{(2) OpenEdition}

Journals

Édition électronique

URL : http://journals.openedition.org/lidil/3196

DOI : 10.4000/lidil.3196

ISSN : 1960-6052

Éditeur

UGA Éditions/Université Grenoble Alpes

Édition imprimée

Date de publication : 15 mai 2012

Pagination : 93-105

ISBN : 978-2-84310-226-4

ISSN : $1146-6480$

Référence électronique

Véronique Leclercq et Anne Vicher, «Étude sur les usages des référentiels dans la formation

linguistique de base », Lidil [En ligne], 45 | 2012, mis en ligne le 15 novembre 2013, consulté le 20 avri 2019. URL : http://journals.openedition.org/lidil/3196 ; DOI : 10.4000/lidil.3196

(c) Lidil 


\title{
Étude sur les usages des référentiels dans la formation linguistique de base
}

\author{
Véronique Leclercq* et Anne Vicher**
}

\begin{abstract}
RÉSUMÉ
Cet article restitue les principaux résultats d'une enquête par questionnaires portant sur les usages des référentiels du domaine langagier par des formateurs impliqués dans la formation linguistique de base d'adultes et jeunes adultes. L'étude montre l'émergence d'une véritable culture professionnelle autour des référentiels de formation, envisagée selon plusieurs angles : l'étendue des connaissances des supports existants, les modalités d'appropriation, les capacités de recul critique et la prise de conscience des enjeux institutionnels.
\end{abstract}

\section{ABSTRACT}

This article takes into account the main results of an inquiry into adult trainers teaching french language and the basic skills focused on the use of linguistic standards in their practice. The results based on the analysis of closed and open questions show a real professional culture: trainers know many different standards and frames of reference and how to use them. Adult trainers are also able to have a critical view on the different instruments and are conscious of institutional issues concerning the matter.

\section{Introduction}

Les pratiques des formateurs peuvent se définir comme l'ensemble des comportements et des actions concrètes menées face à un groupe de

* Professeur de Sciences de l'éducation, Université de Lille 1 - Cueep ; laboratoire CIREL, équipe Trigone.

** Professeur associé, Université de Paris Ouest-Nanterre-La Défense et directrice d'Ecrimed. 
stagiaires, mais aussi les procédés de mise en œuvre de l'activité, les opérations de planification de l'action éducative, les choix effectués et les prises de décision (Altet, 2003). Les usages de supports de cours et de documents qui servent de référence pour l'intervention éducative constituent une des dimensions à interroger quand on cherche à décrire et analyser ces pratiques. Parmi ces supports, les référentiels occupent une place spécifique, notamment parce qu'ils constituent des instruments de la sélection et de la hiérarchisation des savoirs «jugés bons » à être enseignés. La formation de base des jeunes adultes et des adultes n'a pas échappé à la montée en puissance de cette tendance à la « référentialisation » (Chauvigné et Lenoir, 2010 : 9), représentative d'un effort de rationalisation et de cadrage. Même si un certain retard dans le recours à ces approches caractérise le secteur de la formation de base des populations peu scolarisées et peu qualifiées, par rapport à l'ensemble de la formation d'adultes (Leclercq, 2007), ces référentiels, et notamment les référentiels de communication en français, font actuellement partie des ressources disponibles, voire prescrites, pour la conception et la mise en place des actions ${ }^{1}$.

Notre étude vise à interroger les usages que font les intervenants, formateurs ou coordinateurs pédagogiques, des référentiels du domaine langagier, à saisir les représentations qu'ils en ont et les évolutions récentes vécues dans ce domaine. Nous avons basé notre investigation sur une enquête par questionnaires, envoyés en 2011 aux formateurs et coordinateurs pédagogiques intervenant dans les dispositifs d'apprentissage ou de réapprentissage de la lecture-écriture, de formation linguistique des migrants et de remise à niveau en français et autres savoirs de base : actions financées par les Conseils régionaux; par les conseils généraux; par les municipalités; par l'État, à savoir l'Office français de l'immigration et de l'intégration (OFII), l'Agence pour la cohésion sociale et l'égalité des chances, (ACSE), les directions régionales des entreprises, de la concurrence, de la consommation, du travail et de l'emploi (DIRECCTE) ; par les entreprises via les organismes paritaires collecteurs agréés (OPCA). Les régions concernées sont le Nord-

1. Les réformes de la formation linguistique des migrants valorisent la notion de parcours de certification basé sur la validation de compétences formalisées dans les référentiels. Par ailleurs les harmonisations européennes en matière de formation tout au long de la vie incitent à la mise en place de dispositifs portant sur le développement de compétences clés, notamment en langue, décrites dans des référentiels communément partagés. 
Pas-de-Calais (désormais NPDC), l'Ile-de-France, le Midi-Pyrénées, le Languedoc Roussillon et la Bretagne.

$\mathrm{Au}$ total 8 hommes et 56 femmes ont répondu au questionnaire (sur 100 questionnaires envoyés). Ce sont des formateurs impliqués dans le face à face pédagogique direct ( $\mathrm{N}: 32$ dont 10 bénévoles) ou des coordinateurs pédagogiques associant le plus souvent activités d'ingénierie pédagogique et face à face pédagogique (N : 24). Nous avons aussi sollicité le point de vue de quelques formateurs-stagiaires, étudiants de diplômes professionnels $(\mathrm{N}: 6)$ et de professionnels autres $(\mathrm{N}: 2)$. Ces intervenants travaillent dans le secteur associatif, dans le secteur marchand, dans des sociétés coopératives, à l'Éducation nationale et dans des centres sociaux. Nous avons pris garde à varier le panel des enquêtés en termes d'âge et d'ancienneté. Notons que $60 \%$ de l'ensemble ont plus de 10 ans d'ancienneté.

Dans le cadre de cet article, nous ne pouvons traiter l'ensemble des réponses aux questions posées et nous nous centrons sur deux points essentiels articulés entre eux.

Le premier axe d'investigation porte sur la notion même de référentiel de formation du domaine langagier. Après avoir rappelé le caractère multiforme des référentiels, nous analyserons les modalités d'appropriation de ces supports par les praticiens à travers ce qu'ils en disent, leurs conceptions de ce qu'est un référentiel de formation du domaine langagier, mais aussi leurs usages d'une terminologie spécialisée. Le second volet de la recherche part du constat que chaque référentiel est un construit social à situer dans un contexte précis. L'analyse des phases de conception et de diffusion des référentiels nécessite d'interroger les formes de cadrage sous jacentes, les écarts entre le curriculum prescrit et le curriculum réel, les modes de sélection de ce qui est à enseigner, les enjeux des acteurs concernés. Cette analyse n'est pas l'objet de cette contribution, néanmoins notre enquête apporte des éléments de connaissance sur ces points. À travers les questionnaires, seront mis en évidence les choix des référentiels utilisés, les usages concrets et les écarts possibles entre les orientations des concepteurs et les modes d'utilisation sur le terrain, les situations de rejet ou d'adhésion, les appréciations portées sur ces supports par les formateurs, les fonctions attribuées à ces ressources pédagogiques. Répondre à ces différentes questions amène finalement à analyser l'émergence d'une culture professionnelle partagée autour de l'usage des référentiels : étendue des connaissances des ressources, modalités d'appropriation des fonctions des supports, capacités de recul critique, prise de conscience des enjeux institutionnels. 


\section{Autour de la notion de « référentiel »}

\section{Multiplicité des formes de référentiels}

Au cours des dernières décennies, différents types de supports portant le nom de « référentiel » ont été diffusés et utilisés dans le domaine de la formation continue des adultes. Tout en partageant la même dénomination, ils ne sont pas pour autant identiques : les fonctions, les méthodologies de conception, la terminologie, les cadres théoriques de référence peuvent se différencier.

On distingue assez clairement les référentiels métier et de formation. Mais les frontières entre référentiels de formation, d'évaluation, de certification, de compétences sont assez poreuses. Pour ce qui concerne spécifiquement les référentiels du domaine langagier utilisés dans la formation des publics de faible niveau de scolarisation, maitrisant mal la communication orale et écrite, on retrouve cette même ambigüité autour du terme « référentiel ». Dans le recensement effectué par Anne Vicher (2005) sont répertoriés des supports appelés « référentiels de formation », mais aussi des « référentiels d'évaluation » et des « référentiels de description de compétences », ces deux dernières catégories ne portant pas toujours le nom de référentiel. Sont exclus du recensement les simples supports d'évaluation : tests, grilles d'entretien, etc. Le référentiel de formation est défini comme un support qui répertorie et formalise les compétences ou capacités attendues à la fin de séquences de formation, qui les situe sur une échelle ou les gradue selon les niveaux de maitrise et qui mentionne les critères d'évaluation ${ }^{2}$. Les référentiels d'évaluation se différencient dans la mesure où ils se polarisent davantage sur les critères d'évaluation et les indicateurs de réussite et donnent des exemples de tests. Enfin il existe ce qu'on peut appeler des référentiels de compétences à visée descriptive, qui énoncent de façon très

2. Le Référentiel de formation linguistique de base (RFLB, $1^{\mathrm{re}}$ édition $1990,2^{\mathrm{e}}$ édition 1996) est représentatif de ce type de référentiel de formation. Plus récemment conçu, Le Niveau A1.1 pour le français. Référentiel pour les premiers acquis en français. (Beacco et al., 2005) est à la fois référentiel de formation et de certification puisqu'il a été conçu pour valider un premier niveau de maitrise de la communication orale et écrite : le DILF (Diplôme initial de langue française) reconnu institutionnellement dans le cadre des formations linguistiques financées par l'Office français de l'immigration et de l'intégration. 
générale ce qu'un sujet doit être capable de faire pour être jugé « compétent ». Ces supports ont une fonction de cadrage global, fournissent des repères pour la définition de politiques de formation, donnent une direction dans laquelle doit se dérouler l'intervention éducative et précisent davantage les finalités des formations que les objectifs précis. Le terme "cadre de référence » convient davantage dans ce cas de figure que celui de « référentiel ${ }^{3}$ ». À noter que les concepteurs eux-mêmes ne sont pas toujours au clair sur ces termes. Ainsi le Cadre européen commun de référence pour les langues, désormais CECR (2001) a toutes les caractéristiques d'un référentiel de formation.

Notre investigation prend en compte la diversité de cette notion de référentiel, elle cherche à analyser les conceptions des praticiens de ce qu'ils considèrent comme référentiel de formation dans le domaine langagier. Aucune définition standard ne leur a été proposée. Des questions ouvertes permettent de connaitre l'étendue des connaissances des intervenants, d'analyser la façon dont les praticiens appréhendent des supports proches et de percevoir les distinctions qu'ils effectuent ou non entre différents types de ressources.

\section{Connaissances de ressources existantes}

Tous les formateurs, sauf un, répondent à la question : « citez les noms des référentiels du domaine langagier que vous connaissez ». Ils en connaissent beaucoup ( $\mathrm{N}: 30$ ), y compris des référentiels étrangers ou des référentiels très spécialisés (par exemple, le Référentiel d'évaluation linguistique pour les métiers de la propreté, 2009), mais plus de la moitié ne sont cités qu'une seule fois ( $\mathrm{N}: 18)$. Deux référentiels cumulent les occurrences : le Référentiel de formation linguistique de base, désormais $R F L B$, est cité 45 fois sur 64 avec une surreprésentation en NPDC (31 sur 32 réponses) et des appellations diverses et variées. Le CECR l'est 34 fois avec une sous-représentation en NPDC (6 sur 32 contre 28 sur 32 dans les autres régions, dans lesquelles les enquêtés utilisent tous le sigle $C E C R$ ). On peut être surpris par le fait que les

3. Voir par exemple le Cadre national de référence de l'ANLCI (2003) qui décrit en quelques pages quatre degrés d'appropriation des savoirs de base, dont ceux liés à la maitrise du français ou le Cadre de référence européen pour les compétences clés, document de six pages annexé au texte de Recommandation du Parlement européen et du Conseil du 18-12-2006 sur les compétences clés et la formation tout au long de la vie. 
référentiels de niveaux du $C E C R$ pour la langue française soient assez peu répertoriés : le référentiel A1.1 qui apparait parfois sous la dénomination « référentiel DILF», est connu de 11 enquêtés. Le Référentiel de niveau $A 1$ est cité 7 fois, de niveau A2 4 fois et du niveau B1 une seule fois. Deux autres référentiels du domaine langagier sont cités plusieurs fois (N : 6) : Démarche pour l'évaluation (1996 et 2003) et ce qui est appelé le "référentiel québécois », dont le nom exact est maintenant Les niveaux de compétence langagière : accueil et francisation (2009).

En grande majorité, les formateurs ne confondent pas référentiels et supports de cours ou « méthodes » (seuls 4 enquêtés effectuent cette confusion). Plus de la moitié des enquêtés (35 sur 64) perçoit des différences entre types de référentiels et propose des oppositions pertinentes : référentiel linguistique/référentiel professionnel - référentiel de langue/référentiel de savoirs de base - référentiel emploi/référentiel de formation, etc. Ces premiers constats révèlent donc un bon niveau de connaissances des ressources existantes, même si cela porte sur quelques référentiels «dominants » et témoignent d'une appropriation de la notion de référentiel, pour une bonne partie du panel.

Mais on note aussi quelques approximations dans les façons de définir et de catégoriser les ressources. Ainsi le Référentiel des compétences clés en situation professionnelle (2010), désormais RCCSP, est cité 14 fois alors qu'il ne concerne qu'en partie les compétences en langue ( 2 «savoirs » sur les 10 répertoriés). De même, les « cadres de référence » qui ne constituent pas à proprement parler des référentiels de formation sont cependant répertoriés dans cette catégorie. Le Cadre national de référence ANLCI (2003) et le Cadre de référence européen pour les compétences clés (2006) sont cités respectivement 7 et 5 fois. Enfin des référentiels comme le Référentiel du certificat de formation générale (2006) (N : 3) ou le Référentiel des savoirs de base (2000) (N : 5) apparaissent dans la rubrique du domaine langagier, alors qu'il s'agit de documents englobant d'autres champs de savoirs. Les différences domaine langagier / autres domaines ne sont pas toujours finement perçues. Le même support peut être indifféremment cité dans ces deux rubriques ${ }^{4}$. On constate également des confusions terminologiques; les appellations sont parfois ambigües ou approximatives : «Référentiel $A N P E »(\mathrm{~N}: 1)$ - «Référentiel Horizon » $(\mathrm{N}: 1)$ - «Référentiel PJJ»

4. C'est le cas du Référentiel des compétences clés en situation professionnelle, $\mathrm{du}$ Référentiel des savoirs de base et du Cadre national de référence ANLCI répartis pour moitié dans chacune des deux catégories. 
$(\mathrm{N}: 1)$ - «Référentiel européen ». Comment retrouver le support dont il est question?

Le NPDC (32 enquêtés) se différencie des autres régions. Le Cadre national de référence de l'ANLCI n'est jamais cité dans le NPDC et le $C E C R$ y est beaucoup moins connu. À l'opposé le RFLB et le Cadre de référence européen pour les compétences clés y sont davantage répertoriés. La bonne connaissance du RFLB s'explique par le fait que ce support a été conçu et expérimenté dans le NPDC. Les formations consacrées à son usage et la diffusion massive ont contribué à la surutilisation dans cette région. Le Cadre européen, quant à lui, a été promu à partir de 2009 avec la mise en place des dispositifs d'accès aux compétences clés par le conseil régional et par l'État.

Les formateurs ayant plus de 10 ans d'ancienneté connaissent davantage les référentiels du domaine langagier : ils sont $40 \%$ à en citer 3 et plus de 3, alors que ceux ayant moins de 10 ans d'ancienneté ne sont que $12 \%$ à atteindre ce score.

\section{Les référentiels du domaine langagier en formation de base : du construit social aux pratiques des intervenants}

\section{Un construit social}

Les référentiels assument une fonction sociale de régulation entre différents acteurs, «ils manifestent des compromis portant sur les objectifs, les contenus, les modalités et l'évaluation des apprentissages » (Chauvigné, 2010 : 78). On doit souligner l'aspect construit de ces supports, dont la conception et la diffusion sont le résultat de décisions à situer dans un contexte précis. Pour ce qui concerne le domaine de la formation de base de publics maitrisant mal la communication orale et écrite, les phases de conception et d'usages des référentiels s'interprètent au vu des évolutions institutionnelles et pédagogiques de ce secteur. Les politiques de lutte contre l'illettrisme, de formation linguistique des migrants (Adami et Leclercq, 2012) et d'accompagnement à l'insertion de populations «en difficulté » ont été marquées par des transformations importantes depuis les années 1990 associant une tendance à la normalisation et à l'homogénéisation des pratiques, censées améliorer et optimiser l'offre de formation, à une volonté de cadrage et de prescription des financeurs et des pouvoirs publics. Ces deux lignes de force, proches mais distinctes, $\mathrm{s}$ 'articulent à certaines périodes pour constituer un terrain favorable à la référentialisation. 
Analyser les contextes de conception et de diffusion des référentiels nécessite, en tout état de cause, d'interroger le rôle des instances décisionnelles et des acteurs dans la sélection des programmes et de répondre à l'interrogation « qui contrôle quoi ? ». Cela concerne aussi la question de la fonction des chercheurs et experts dans la conception des outils de cadrage. Les conditions de l'élaboration de ces supports révèlent des tensions entre des territoires d'expertise. Enfin il est pertinent de s'intéresser au passage d'une prescription institutionnelle descendante à une mise en pratique sur les terrains et aux tensions entre curriculum prescrit et curriculum réel. C'est essentiellement à cet aspect que nous nous intéresserons dans l'analyse des réponses aux questionnaires. Que deviennent ces outils de cadrage sur les terrains de formation? Lesquels sont les plus utilisés? Quels en sont les usages? Dans quel contexte (dispositifs et publics)? Avec quelles appréciations des usagers? Quelles connaissances des contextes institutionnels?

\section{Les usages des référentiels}

Plus de la moitié des enquêtés ont en leur possession un ou plusieurs référentiels, ce qui constitue un indicateur de proximité.

$80 \%$ du panel utilisent un ou des référentiel(s) du domaine langagier dans leurs actions. Les deux référentiels les plus utilisés sont à nouveau le $R F L B$, répertorié par $67 \%$ des utilisateurs et le CECR par $38 \%$. Le $R F L B$ est à nouveau surreprésenté dans le NPDC (utilisé à $86 \%$ contre $43 \%$ dans les autres régions). De plus il est une ressource exclusive dans $60 \%$ des cas, alors que dans les autres régions il est utilisé en complément d'autres supports, notamment du CECR. À l'opposé ce dernier référentiel est peu utilisé dans le NPDC (14\%) comparé aux autres lieux (70\%). Le spectre d'usage du RFLB et du CECR est large : dispositifs régionaux de réapprentissage des savoirs de base - cours municipaux de la ville de Paris - programme « compétences clés » financé par l'État - cours d'alphabétisation des quartiers. Au contraire le RCCSP n'est exploité qu'en entreprises ( $\mathrm{N}: 7)$ et l'usage du Référentiel Al.1 (N : 5) se cantonne très majoritairement aux actions OFII en association avec le $R F L B$ ou le $C E C R^{5}$. Tous les autres référentiels ont un très faible taux d'occurrence $(\mathrm{N}: 1$ ou 2$)$.

5. Intervenir dans le cadre des actions de l'OFII ne signifie pas obligatoirement utiliser le $A 1.1$ ou les autres référentiels de niveaux $A 1, A 2$. En effet sur les 12 intervenants « $\mathrm{OFII} », 8$ utilisent d'autres référentiels que le A1.1. Lorsque 
Trois pôles d'usages sont à distinguer : les pôles pédagogie, communication et management de la formation.

Le premier pôle fait référence aux usages :

- soit dans la définition et la formalisation des compétences attendues et des objectifs de formation (items : « décliner les objectifs »- « décrire les compétences », etc.);

- soit dans la planification pédagogique, la préparation des programmes et des séances, la définition de parcours (items : «établir des plans de travail »- « construire les contenus », « élaborer une progression », etc.);

- soit dans l'évaluation des apprenants (items : "positionner» - « repérer les acquis » - « repérer les niveaux »- « faciliter l'orientation », etc.);

- soit dans la conception de supports (items : « concevoir les supports »- « imaginer des exercices », etc.).

Le second pôle renvoie au référentiel comme instrument de communication entre partenaires (items : « annoncer le projet aux stagiaires »« informer sur ce qu'on va faire », etc.).

Le troisième concerne le référentiel comme aide au management institutionnel de la formation et au cadrage des actions (items : « avoir une vision partagée de la formation $»-$ « donner un cadre »- « répondre aux exigences des financeurs »- « harmoniser les pratiques »- « répondre aux appels d'offre », etc.).

À la question concernant les fonctions générales d'un référentiel, le pôle largement dominant est le pôle pédagogie avec 120 items de ce type répertoriés. Ce sont les versants «planification pédagogique » et «évaluation » qui sont les plus représentés $(\mathrm{N}: 42, \mathrm{~N}: 40)$, puis le versant «définition des objectifs » $(\mathrm{N}: 33)$. La conception de supports est peu citée $(\mathrm{N}: 5)$. Le référentiel apparait de façon moins marquée comme aide au management $(\mathrm{N}: 18)$, puis de façon marginale comme instrument de communication $(\mathrm{N}: 6)$.

À la question des usages concrets des référentiels du domaine langagier dans leurs actions, les enquêtés utilisateurs $(\mathrm{N}: 52)$ répondent là encore massivement à l'aide d'items du pôle pédagogie avec une

les formateurs ont affaire à des groupes d'étrangers peu ou pas scolarisés, ils ont recours bien davantage au $R F L B$ qu'au référentiel A1.1 en l'associant parfois au CECR (56\% des cas). 
surreprésentation du versant évaluation (34 items) et du versant planification (36 items), les 2 autres versants sont plus marginalement cités $(\mathrm{N}: 15$ et $\mathrm{N}: 3)$. Le référentiel est aussi vu comme outil de management (14 items) et peu envisagé comme outil de communication (4 items).

Les référentiels sont donc appréhendés avant tout en tant qu'outils opérationnels pour l'action pédagogique, notamment pour la planification et l'évaluation des apprenants, ce qui correspond tout à fait aux fonctions envisagées par les concepteurs.

\section{Connaissance des enjeux institutionnels}

Sur 52 enquêtés utilisant des référentiels, 28 se disent «obligés », 11 font allusion à une prescription institutionnelle («C'est indiqué dans le cahier des charges », « les financeurs nous obligent », etc.) et 17 à une incitation pédagogique de l'organisme, du réseau de coordination des actions ou à une pression auto-administrée. La prescription institutionnelle semble donc faible et ne concerne que $20 \%$ des utilisateurs.

$70 \%$ des intervenants savent si l'usage des référentiels est obligatoire et sont capables de citer quel(s) référentiel(s) sont obligatoires et pour quels dispositifs et cahiers des charges ou si aucun n'est obligatoire. Ceux qui méconnaissent l'environnement institutionnel sont tous vacataires, formateurs à temps partiel, bénévoles, apprentis formateurs, mais ne sont pas forcément des novices dans le secteur.

Enfin les enjeux institutionnels s'envisagent aussi du côté des modes d'appropriation formelle et organisée des ressources. $60 \%$ des utilisateurs ont suivi une formation ou une sensibilisation à l'utilisation de référentiels et $43 \%$ participent à des réunions d'équipes internes sur cette question, celles-ci s'avérant plus nombreuses dans le NPDC que dans les autres régions (15/29 contre 7/23).

\section{Appropriation critique}

On compte davantage d'items d'appréciations positives que négatives. La question ouverte concernait les défauts et qualités des référentiels utilisés. Il y a un taux de non réponses assez important surtout pour la question des défauts ( 19 sur 52 ). Les items positifs, tels que « donner un langage commun », «c'est un guide », «permet de concevoir des tests », « cela rend explicite les étapes », etc. représentent environ 65 citations différentes, alors que les items négatifs, tels que « cela peut être enfermant », « c'est hermétique », " c'est une usine à gaz », « c'est trop compliqué, trop théorique », etc. en représentent 50. Les avis sont assez 
contradictoires, les référentiels sont en même temps jugés « simples d'accès » et « complexes », « détaillés » et « manquant d'exemples », « formalisant des étapes claires » et « trop hiérarchisés ». Il est très difficile de voir émerger des tendances claires sur ce point. Au final, ces appréciations montrent que les formateurs se sont confrontés à une appropriation concrète de ces ressources et qu'ils sont capables d'un regard critique.

Par ailleurs, les formateurs ont une conscience claire des usages des référentiels et de leurs limites. Les non utilisateurs de référentiels (19\% du panel) ont recours à d'autres ressources proches (ouvrages de vulgarisation, méthodes de langue, portfolios, grilles d'évaluation), exploitées également par les utilisateurs, qui savent que les référentiels ne suffisent pas. 50 types de ressources différentes sont cités : 15/50 sont des outils ou grilles d'évaluation ou de compétences provenant de supports ou de manuels de présentation d'examen. 15/50 concernent le portfolio Mon livret d'apprentissage du français. Paris sur le français (Vicher, 2008). Viennent ensuite les «tableaux d'objectifs et de contenus » de méthodes de langue (15/50). 5 formateurs citent le RCCSP, qu'ils ne perçoivent pas comme un référentiel.

\section{Conclusion : synthèse des résultats}

L'analyse d'un certain nombre de résultats de l'enquête montre qu'émerge une véritable culture professionnelle autour des usages des référentiels. Les intervenants les connaissent et les utilisent. Ils les différencient d'autres ressources (méthodes, portfolios, grilles d'évaluation, etc.), même si des approximations persistent dans les catégorisations fines et les terminologies, ce qui peut se comprendre au vu du « sémantisme flottant 》 (Cros et Raisky, 2010 :112) associé à la notion de référentiel. Ils perçoivent clairement les fonctions des supports, les utilisent dans le sens envisagé par les concepteurs et les complètent judicieusement par d'autres aides pédagogiques. Les enquêtés sont capables de recul critique. Tout en les jugeant « lourds », « pas assez pratiques », « complexes », ils les considèrent informatifs et nécessaires au cadrage de l'action de formation. Ils en acceptent la rigueur, tout en gardant une certaine liberté dans les applications pratiques.

Les contextes et lieux de conception et diffusion des référentiels ont un impact sur les usages concrets et expliquent quelques différences 
constatées entre régions et entre types de dispositifs concernés ${ }^{6}$. Les usagers des référentiels ont en majorité une connaissance des prescriptions institutionnelles, même si l'on constate une différence entre les formateurs-coordonnateurs à temps plein et les vacataires ou bénévoles à temps partiel.

\section{RÉFÉRENCES BIBLIOGRAPHIQUES}

AdAmi H. et LeCLeRCQ V. (éds) (2012) : Les migrants face aux langues des pays d'accueil : acquisition en milieu naturel et formation, Villeneuve d'Ascq, Presses universitaires du Septentrion.

Altet M. (2003) : «Caractériser, expliquer et comprendre les pratiques enseignantes pour aussi contribuer à leur évaluation », Les dossiers des sciences de l'éducation, $\mathrm{n}^{\circ} 10$, p. 31-43.

Chauvigné C. (2010) : «Les référentiels en formation », Recherche et Formation, $\mathrm{n}^{\circ}$ 64, p. 77-90.

Chauvigné C. et Lenoir Y. (2010) : «Les référentiels en formation : enjeux, légitimité, contenu et usage », Recherche et Formation, n ${ }^{\circ}$ 64, p. 9-14.

Cros F. et RaISKy C. (2010) : «Autour des mots de la formation : référentiel », Recherche et Formation, $n^{\circ}$ 64, p. 105-116.

LECLERCQ V. (2007) : «La formation de base : publics, dispositifs, pratiques », Savoirs, $\mathrm{n}^{\circ} 14$, p. 11-55.

Vicher A. (2005) : Référentiels et outils d'évaluation des compétences de base, Les dossiers de l'ANLCI, Lyon, ANLCI.

\section{RÉFÉRENTIELS, CADRES DE RÉFÉRENCE ET RESSOURCES CITÉS}

Beacco J.-C., de Ferrari M., Lhote G. et Tagliante C. (2005) : Niveau A1.1 pour le français. Référentiel et certification (Dilf) pour les premiers acquis en français, Paris, Didier.

Cadre européen commun de référence pour les langues, Direction des politiques linguistiques, Conseil de l'Europe, Paris, Didier, 2001.

6. Le RFLB, conçu dans le NPDC et prescrit dans les appels d'offres du conseil régional, y est plus largement utilisé. De même le RCCSP, créé dans le cadre de partenariats avec des OPCA, est largement référencé par les formateurs intervenant en entreprise. On peut aussi citer la fréquente utilisation en Îlede-France du portfolio élaboré pour la mairie de Paris (Vicher, 2008). Remarquons aussi l'utilisation exclusive du référentiel DILF dans les formations de l'OFII, même si il est concurrencé par des supports plus anciens. 
Cadre national de référence, Lyon, Agence Nationale de Lutte contre 1'Illettrisme, 2003.

Cadre de référence européen pour les compétences clés, annexe à la recommandation du Parlement européen et du Conseil sur les compétences clés pour l'éducation et la formation tout au long de la vie, Strasbourg, Journal officiel de l'Union européenne, 2006.

Certification initiale de compétences en français, Centre de linguistique de Besançon, université de Franche Comté, 2003.

Démarche pour l'évaluation, des niveaux débutants aux niveaux intermédiaires en français langue étrangère, Paris, Cimade, 1996 et 2003.

Les niveaux de compétence langagière : accueil et francisation, Adaptation du matériel de l'Immigration et des communautés culturelles du Québec, Québec, 2009.

Référentiel de formation linguistique de base, Lille, Cueep-Fas-Dafco, 1990 et 1996.

Référentiel des savoirs de base, dans C. Dartois et C. Thiry, Former les publics peu qualifiés, ministère Emploi et Solidarité, 2000.

Référentiel d'évaluation linguistique pour les métiers de la propreté, Groupe Langage, Travail et Formation du CRAPEL /ATILF, Nancy 2, 2009.

Référentiel du certificat de formation générale en français et maths, Éducation nationale, 2006.

Référentiel de compétences clés en situation professionnelle, Lyon, Agence Nationale de Lutte contre l'Illettrisme, 2010.

VICHER A. (coord.) (2008) : Mon livret d'apprentissage du français. Paris sur le français, Mairie de Paris. 
